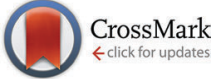

Cite this: Phys. Chem. Chem. Phys., 2014, 16, 22995

Received 16th August 2014, Accepted 8th September 2014 DOI: $10.1039 / c 4 c p 03669 g$

www.rsc.org/pccp

\section{Hydrogen-bond memory and water-skin supersolidity resolving the Mpemba paradox}

\author{
Xi Zhang, ${ }^{* a b}$ Yongli Huang, ${ }^{* c}$ Zengsheng Ma, ${ }^{c}$ Yichun Zhou, $^{c}$ Ji Zhou, ${ }^{d}$ \\ Weitao Zheng, ${ }^{e}$ Qing Jiang ${ }^{e}$ and Chang Q. Sun $\star^{* a}$
}

\begin{abstract}
The Mpemba paradox, that is, hotter water freezes faster than colder water, has baffled thinkers like Francis Bacon, René Descartes, and Aristotle since B.C. 350. However, a commonly accepted understanding or theoretical reproduction of this effect remains challenging. Numerical reproduction of observations, shown herewith, confirms that water skin supersolidity [Zhang et al., Phys. Chem. Chem. Phys., DOI: 10.1039/C1034CP02516D] enhances the local thermal diffusivity favoring heat flowing outwardly in the liquid path. Analysis of experimental database reveals that the hydrogen bond $(\mathrm{O}: \mathrm{H}-\mathrm{O})$ possesses memory to emit energy at a rate depending on its initial storage. Unlike other usual materials that lengthen and soften all bonds when they absorb thermal energy, water performs abnormally under heating to lengthen the $\mathrm{O}: \mathrm{H}$ nonbond and shorten the $\mathrm{H}-\mathrm{O}$ covalent bond through inter-oxygen Coulomb coupling [Sun et al., J. Phys. Chem. Lett., 2013, 4, 3238]. Cooling does the opposite to release energy, like releasing a coupled pair of bungees, at a rate of history dependence. Being sensitive to the source volume, skin radiation, and the drain temperature, the Mpemba effect proceeds only in the strictly non-adiabatic 'source-path-drain' cycling system for the heat "emission-conduction-dissipation" dynamics with a relaxation time that drops exponentially with the rise of the initial temperature of the liquid source.
\end{abstract}

\section{Introduction}

A commonly accepted explanation or numerical reproduction of the Mpemba paradox remains challenging despite efforts made since the age of Aristotle. ${ }^{1-5}$ Proposed factors explaining this effect include evaporation, ${ }^{6}$ frosting, ${ }^{7}$ solutes, ${ }^{8}$ supercooling, ${ }^{7,9}$ thermal convection, ${ }^{10,11}$ etc. According to the winner ${ }^{12}$ of a competition held in 2012 by the Royal Society of Chemistry, thermal convection rationalizes the energy "emission-conductiondissipation" dynamics in the "source-path-drain" system in which the Mpemba paradox takes place. However, little attention has yet been paid to the intrinsic nature and the relaxation dynamics of the hydrogen bond $(\mathrm{O}: \mathrm{H}-\mathrm{O})^{13}$ as the primary component of the liquid source for heat emission and the liquid path for heat conduction. In this communication, we show

\footnotetext{
${ }^{a}$ NOVITAS, School of Electrical and Electronic Engineering, Nanyang Technological University, Singapore 639798. E-mail: ecqsun@ntu.edu.sg

${ }^{b}$ Center for Coordination Bond and Electronic Engineering, College of Materials Science and Engineering, China Jiliang University, Hangzhou 310018, China ${ }^{c}$ Key Laboratory of Low-Dimensional Materials and Application Technologies (Ministry of Education) and Faculty of Materials, Optoelectronics and Physics, Xiangtan University, Hunan 411105, China

${ }^{d}$ State Key Laboratory of New Ceramics and Fine Processing, Department of Materials Science and Engineering, Tsinghua University, Beijing 100084, China

${ }^{e}$ School of Materials Science, Jilin University, Changchun 130012, China

$\dagger$ Chang Q. Sun has honorary appointments in all affiliations.
}

quantitatively that the $\mathrm{O}: \mathrm{H}-\mathrm{O}$ bond memory and the water-skin supersolidity ${ }^{14,15}$ resolve this paradox with reproduction of the observed attributes. ${ }^{2,12}$

\section{Numerical solution: water-skin supersolidity}

\subsection{Fourier thermal-fluid equation}

We firstly conducted numerical calculation by introducing the skin supersolidity ${ }^{14,15}$ into the path of heat conduction. Molecular under coordination shortens and stiffens the $\mathrm{H}-\mathrm{O}$ bond and meanwhile lengthens and softens the $\mathrm{O}: \mathrm{H}$ nonbond through Coulomb repulsion between electron pairs on adjacent oxygen ions. This process turns the skin of water and ice into the supersolid phase that is elastic, polarized, thermally stable, highly tensile, hydrophobic, and self-lubricant. ${ }^{14,16} \mathrm{~A}$ mass density of $0.75 \mathrm{~g} \mathrm{~cm}^{-3}$, a high-frequency phonon of $3450 \mathrm{~cm}^{-1}$, an $\mathrm{O}$ 1s binding energy of $538.1 \mathrm{eV}$ and a melting point of $315 \mathrm{~K}$ compared to the bulk values listed in Table 1 characterize the skin supersolidity.

The Fourier equation ${ }^{17}$ with appropriate initial-and-boundary conditions best describes the process of thermal-fluid transportation in the liquid water but the skin-supersolidity is necessary. In order to examine all possible factors contributing to the Mpemba effect, we solved this initial-and-boundary condition problem using the finite element calculation method. 


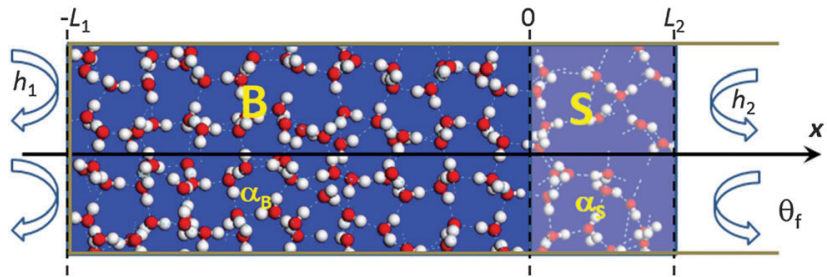

Fig. 1 Water in the adiabatically walled, open-ended, one-dimensional tube cell at initial temperature $\theta_{\mathrm{i}}$ is cooled in the drain of $\theta_{\mathrm{f}}$. The liquid source is divided into the bulk $\left(B\left(-l_{1}=-9 \mathrm{~mm}, 0\right)\right.$ and the skin $\left(S\left(0, l_{2}=1 \mathrm{~mm}\right)\right)$ in the right-hand side) region along the $x$-axis with thermal diffusivity $\alpha_{B}$ and $\alpha_{S}$ and the mass density ratio of $\rho_{\mathrm{S}} / \rho_{\mathrm{B}}=3 / 4^{14,16}$ in the respective region. $x=0$ is the bulk-skin interface. $h_{j}$ is the heat transfer (radiation) coefficient at the tube ends with the absence $(j=1)$ and presence $(j=2)$ of the skin.

Fig. 1 illustrates the adiabatically walled, open-ended, onedimensional tube cell containing water at the initial temperature $\theta_{\mathrm{i}}$. We divide the tube cell into the bulk $\left(\mathrm{B}\right.$, from $-l_{1}=-9 \mathrm{~mm}$ to 0 ) and the skin ( $\mathrm{S}$, from 0 to $l_{2}=1 \mathrm{~mm}$ ) region along the $x$-axis and cool it in the drain of constant temperature $\theta_{\mathrm{f}}$. The $\theta_{\mathrm{f}}$ is subject to variation.

The rate of temperature change in any point $(x)$ of the partitioned tube cell follows the step-function, for simplicity, and the initial-and-boundary conditions:

$$
\begin{aligned}
& \frac{\partial \theta(x)}{\partial t}=\nabla \cdot(\alpha(\theta(x), x) \nabla \theta(x))-v \cdot \nabla \theta(x)
\end{aligned}
$$

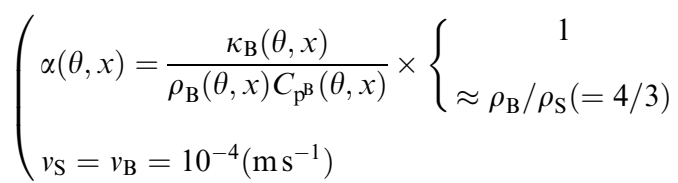

$$
\begin{aligned}
& \begin{cases}\theta=\theta_{\mathrm{i}} & (t=0) \\
\theta\left(0^{-}\right)=\theta\left(0^{+}\right) ; \theta_{x}\left(0^{-}\right)=\theta_{x}\left(0^{+}\right) & (x=0) \\
h_{\mathrm{i}}\left(\theta_{\mathrm{f}}-\theta\right) \pm \kappa_{\mathrm{i}} \theta_{x}=0 & \left(x=-l_{1} ; l_{2}\right)\end{cases}
\end{aligned}
$$

Using a slope function at the interface complicated calculation without changing the physical meanings. The first term describes thermal diffusion and the second thermal convection in the Fourier equation with $\alpha$ being the thermal diffusivity and $v$ the convection rate. The known temperature dependence of the thermal conductivity $\kappa(\theta)$, the mass density $\rho(\theta)$, and the specific heat under constant pressure $C_{\mathrm{p}}(\theta)$, given in Fig. 8 in the Appendix, determine the thermal diffusivity of bulk water $\alpha_{\mathrm{B}}$. The skin supersolidity ${ }^{14}$ contributes to the $\alpha_{\mathrm{S}}$ in the form of $\alpha_{\mathrm{S}}(\theta) \approx 4 / 3 \alpha_{\mathrm{B}}(\theta)$ because the skin mass density $0.75 \mathrm{~g} \mathrm{~cm}^{-3}$ is $3 / 4$ times that of the standard at $4{ }^{\circ} \mathrm{C}$. The $\alpha_{\mathrm{S}}(\theta)$ is subject to optimization as the skin supersolidity may modify the $\kappa(\theta) / C_{\mathrm{p}}(\theta)$ value as well in a yet unknown way.

The boundary conditions represent that at $t>0$, both the temperature $\theta$ and its gradient $\theta_{x}=\partial \theta / \partial x$ continue at the skinbulk interface $(x=0)$ and the thermal flux $h\left(\theta_{\mathrm{f}}-\theta\right)$ are conserved at both ends of the tube. The velocity field of heat convection takes the bulk value of $v_{\mathrm{S}}=v_{\mathrm{B}}=10^{-4}$ or $0 \mathrm{~m} \mathrm{~s}^{-1}$ for examination. As the heat transfer (through radiation) coefficient $h_{j}$ depends linearly on the thermal conductivity $\kappa$ in the respective region, ${ }^{18}$ we took the standard value of $h_{1} / \kappa_{\mathrm{B}}=h_{2} / \kappa_{\mathrm{S}}=30 \mathrm{w} \mathrm{m}^{-2} \mathrm{~K}^{-1}$ (ref. 19) in solving the problem. The $h_{2} / \kappa_{\mathrm{S}}$ term contains the boundary heat reflection that is also negligible. The $h_{2} / h_{1}$ ratio $>1$ describes the possible effect of thermal radiation of the skin.

\subsection{Examination of the thermal convection and diffusivity}

The computer reads in the digitized $\rho(\theta), \kappa(\theta)$, and $C_{\mathrm{p}}(\theta)$ in Fig. 8 to compose the $\alpha_{\mathrm{B}}(\theta)$ before each iteration of calculating the partitioned elemental cells. Besides the thermal diffusivity and the convection velocity field in the Fourier equation, we examined all possible parameters in the initial-and-boundary conditions. Results in Fig. 2 and 3 revealed the following:

(1) Characterized by the crossing temperature of the relaxation $\theta\left(\theta_{\mathrm{i}}, t\right)$ curves, the Mpemba effect occurs only in the presence of the skin supersolidity $\left(\alpha_{\mathrm{S}} / \alpha_{\mathrm{B}}>1\right)$ disregarding the thermal convection.

(2) Complementing the skin supersolidity, thermal convection raises only slightly the skin-bulk temperature difference, $\Delta \theta$, and the crossing temperature.

(3) The Mpemba effect is sensitive to the source volume, the $\alpha_{\mathrm{S}} / \alpha_{\mathrm{B}}$ ratio, the radiation $h_{2}$, and the drain temperature $\theta_{\mathrm{f}}$.

(4) The bulk/skin thickness $\left(l_{1}: l_{2}\right)$ ratio and the thermal convection velocity have little effect on observations.

For instance, increasing the liquid volume may annihilate the Mpemba effect because of the non-adiabatic process of heat dissipation. It is understandable that cooling one drop of $1 \mathrm{~mL}$ water needs shorter time than cooling one cup of $200 \mathrm{~mL}$ water at the same $\theta_{\mathrm{i}}$ under the same conditions. Higher skin radiation $h_{2} / h_{1}>1$ promotes the Mpemba effect. Therefore, conditions for the Mpemba effect are indeed very critical, which explains why the Mpemba effect occurs infrequently.

\subsection{Reproduction of the Mpemba attributes}

Fig. 4 shows numerical reproduction of the observed Mpemba attributes (insets), ${ }^{2,12}$ which confirmed the following:

(1) Hotter water freezes faster than colder water does under the same conditions;

(2) The liquid temperature $\theta$ drops exponentially with cooling time $(t)$ for transiting water into ice with a relaxation time $\tau$ that drops as the $\theta_{\mathrm{i}}$ is increased;

(3) The water skin is warmer than sites inside the liquid and the skin of hotter water is even warmer throughout the course of cooling.

\section{Experimental revelation: $\mathrm{O}: \mathrm{H}-\mathrm{O}$ bond memory}

\section{1 $\theta_{\mathrm{i}}$ dependence of the $\mathrm{H}-\mathrm{O}$ bond linear velocity}

The following formulates the decay curve $\theta\left(\theta_{\mathrm{i}}, t\right)$ shown in Fig. $4 \mathrm{a}^{12}$

$$
\left\{\begin{array}{cl}
\mathrm{d} \theta=-\tau_{\mathrm{i}}^{-1} \theta \mathrm{d} t & \text { (decay function) } \\
\tau_{\mathrm{i}}^{-1}=\sum_{j} \tau_{\mathrm{i} j}^{-1} & \text { (relaxation time) }
\end{array}\right.
$$

The $\theta_{\mathrm{i}}$ dependent relaxation time $\tau_{\mathrm{i}}$ is the sum of $\tau_{j \mathrm{i}}$ over all possible $j$ th processes of heat loss during cooling. 

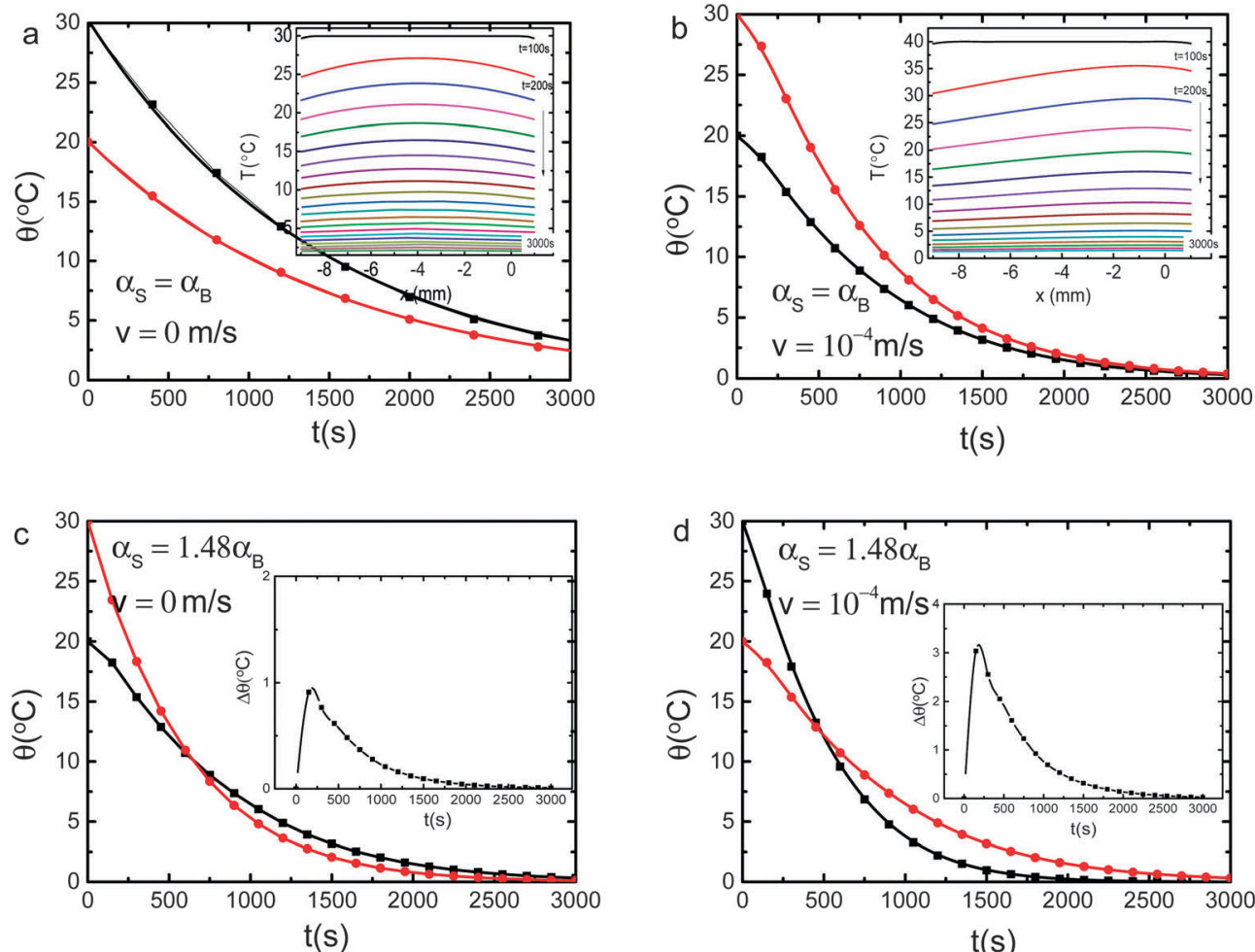

Fig. 2 Thermal relaxation curves $\theta\left(\theta_{i}, t\right)$ (at $x=0$ ) with the $(a, b)$ absence $\left(\alpha_{S} / \alpha_{B}=1\right)$ and $(c, d)$ presence (optimized at $\left.\alpha_{S} / \alpha_{B}=1.48\right)$ of the skin supersolidity and with the $(a, c)$ absence $\left(v_{S}=v_{B}=0\right)$ and $(b, d)$ presence $\left(v_{S}=v_{B}=10^{-4} \mathrm{~m} \mathrm{~s}^{-1}\right)$ of the thermal convection of the liquid heat source. The Mpemba effect characterized by the crossing temperature of the $\theta\left(\theta_{\mathrm{i}}, t\right)$ curves occurs only in the presence of the skin supersolidity disregarding the thermal convection. Insets (a) and (b) show the time dependent thermal-field in the tube cell. Supplementing the skin supersolidity, convection only raises slightly the $\Delta \theta$ and the crossing temperature.

Excitingly, the documented experimental profiles of $\theta\left(\theta_{\mathrm{i}}, t\right)^{12}$ (Fig. 4a) and $d_{\mathrm{H}}(\theta)^{16}$ (Fig. 5a) allow us to show directly the memory of the $\mathrm{O}: \mathrm{H}-\mathrm{O}$ bond without needing any assumption or approximation. The $\theta\left(\theta_{\mathrm{i}}, t\right)$ curve provides the slope of $\mathrm{d} \theta / \mathrm{d} t=-\tau_{\mathrm{i}}^{-1} \theta$ and the $d_{\mathrm{H}}(\theta)=1.0042-2.7912 \times 10^{-5} \exp [(\theta+273) / 57.2887](\AA)^{16}$ curve formulates the measured $\theta$ dependence of the $\mathrm{H}-\mathrm{O}$ bond relaxation. Multiplying slopes of both curves yields immediately the $d_{\mathrm{H}}$ linear velocity under cooling.

The $\mathrm{O}: \mathrm{H}$ nonbond is correlated with the $\mathrm{H}-\mathrm{O}$ bond in relaxation by the equation in Table 1 . As $E_{\mathrm{x}}=k_{\mathrm{x}}\left(\Delta d_{\mathrm{x}}\right)^{2} / 2$ approximates the energy stored in the respective bond with $k_{\mathrm{x}}$ being the force constant, one can obtain the velocities of $d_{\mathrm{x}}$ and $E_{\mathrm{x}}$ readily (x $=\mathrm{L}$ and $\mathrm{H}$ denote the $\mathrm{O}: \mathrm{H}$ and the $\mathrm{H}-\mathrm{O}$ bond, respectively). For simplicity and concise, we will be focused on the instantaneous velocity of $d_{\mathrm{H}}$ during relaxation:

$$
\frac{\mathrm{d}\left(d_{\mathrm{H}}(\theta)\right)}{\mathrm{d} t}=\frac{\mathrm{d}\left(d_{\mathrm{H}}(\theta)\right)}{\mathrm{d} \theta} \frac{\mathrm{d} \theta}{\mathrm{d} t}=-\tau_{\mathrm{i}}^{-1} \theta \frac{\Delta\left(d_{\mathrm{H}}(\theta)\right)}{57.2887}
$$

With $\Delta\left(d_{\mathrm{H}}(\theta)\right)=-2.7912 \times 10^{-5} \exp [(\theta+273) / 57.2887]$

Fig. $5 \mathrm{~b}$ plots the $\theta_{\mathrm{i}}$ dependence of the $d_{\mathrm{H}}$ linear velocity, which confirms that the $\mathrm{O}: \mathrm{H}-\mathrm{O}$ bond indeed possesses memory. Although passing through the same temperature on the way to freezing, the initially shorter $\mathrm{H}-\mathrm{O}$ bond at higher temperature remains highly active compared to those initially longer ones at lower temperatures when they meet on the way of freezing.

\section{2 $\theta_{\mathrm{i}}$ dependence of the relaxation time}

Solving the decay function (2) yields the relaxation time $\tau_{\mathrm{i}}\left(t_{\mathrm{i}}, \theta_{\mathrm{i}}, \theta_{\mathrm{f}}\right)$,

$$
\tau_{\mathrm{i}}=-t_{\mathrm{i}}\left[\operatorname{Ln}\left(\frac{\theta_{\mathrm{f}}+b_{\mathrm{i}}}{\theta_{\mathrm{i}}+b_{\mathrm{i}}}\right)\right]^{-1}
$$

An offset of $\theta_{\mathrm{f}}\left(=0{ }^{\circ} \mathrm{C}\right)$ and $\theta_{\mathrm{i}}$ by a constant $b_{\mathrm{i}}$ is necessary to ensure $\theta_{\mathrm{f}}+b_{\mathrm{i}} \geq 0$ in the solution $\left(b_{\mathrm{i}}=5\right.$ was taken with reference to the fitting in Fig. 4a). With the measured $t_{\mathrm{i}}, \theta_{\mathrm{i}}$, and $\theta_{\mathrm{f}}$, given in Fig. $6 \mathrm{a}$ (scattered data), as input, one can find the respective $\tau_{\mathrm{i}}$ that is featured in the solid line. According to the fitting, $\tau_{\mathrm{i}}$ drops exponentially with the increase of $\theta_{\mathrm{i}}$, or with the increase of the initial energy storage or vibration frequency both of which are experimental results, ${ }^{20}$ as shown in Fig. 6 b.

\section{Discussion: heat "emission- conduction-dissipation"}

\subsection{Liquid source and path: Heat emission and conduction}

The $\mathrm{O}: \mathrm{H}-\mathrm{O}$ bond approximates a pair of asymmetric, coupled, H-bridged oscillators with short-range interactions and memory. ${ }^{21}$ Fig. 7 illustrates interactions and the cooperative relaxation of the $\mathrm{O}: \mathrm{H}-\mathrm{O}$ bond in water under thermal excitation cycling. 

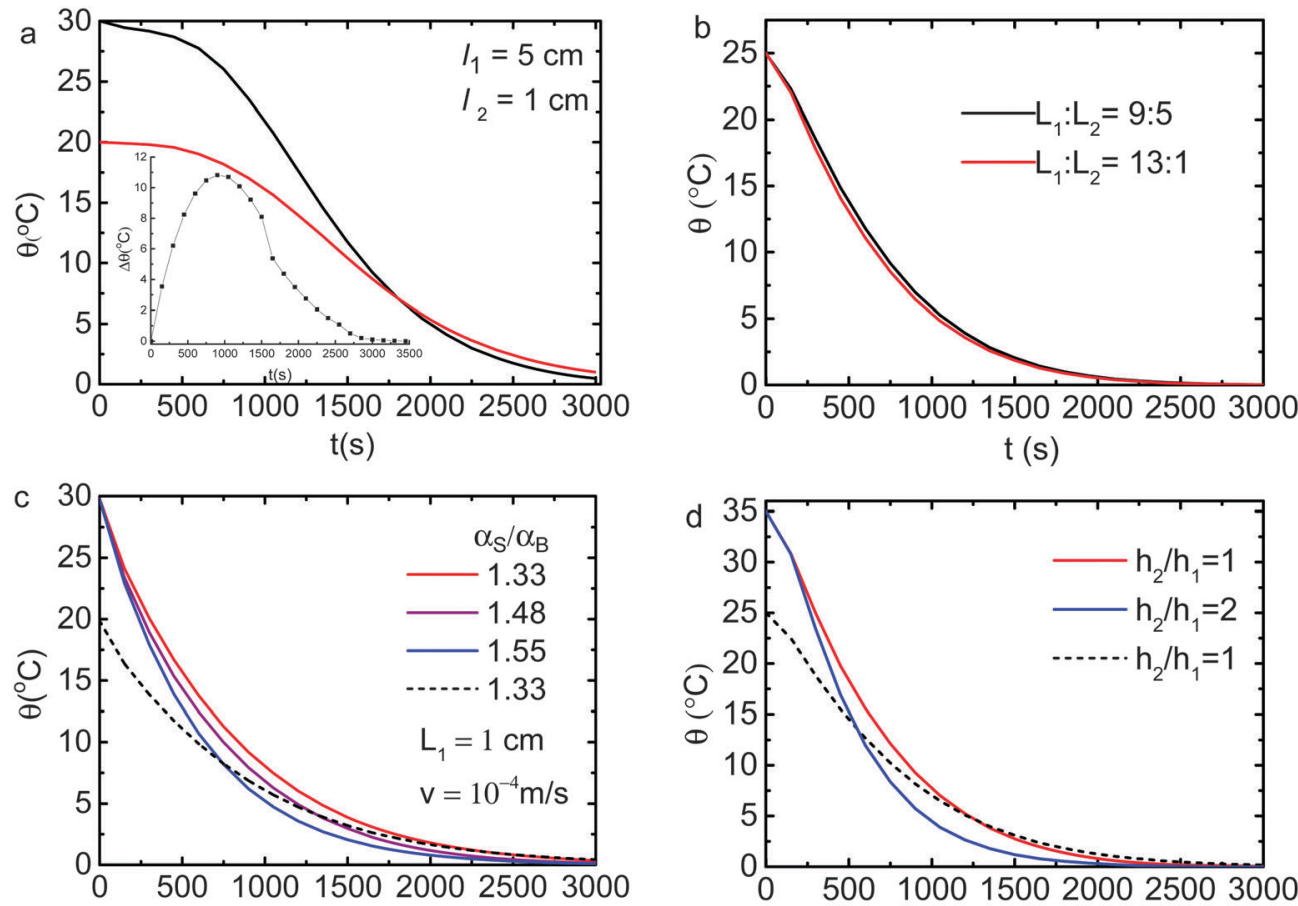

$\mathrm{t}(\mathrm{s})$
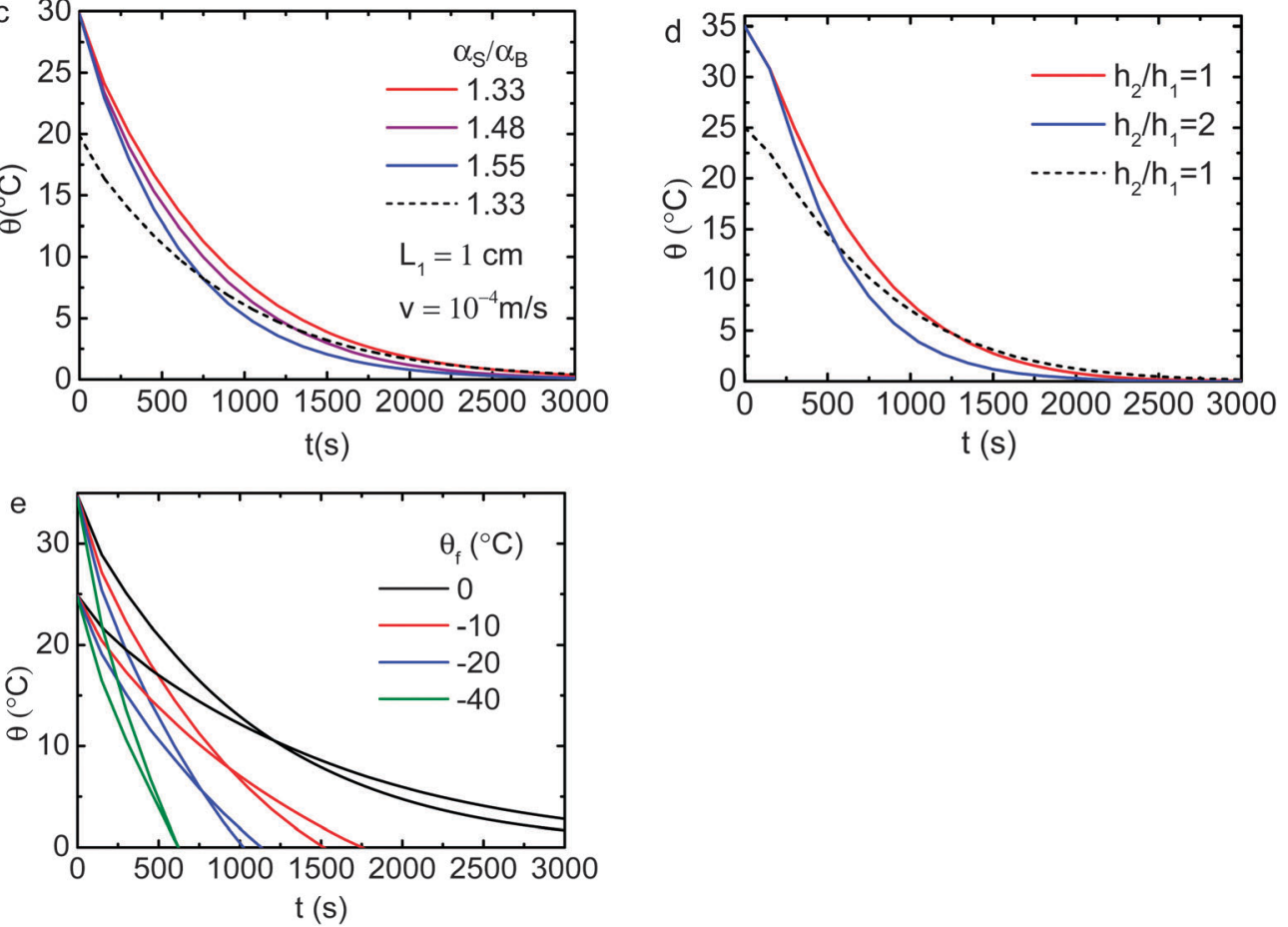

Fig. 3 Sensitivity of the Mpemba effect (crossing temperature) to the (a) source volume, (b) bulk/skin thickness ratio $\left(l_{1}: l_{2}\right)$, (c) $\alpha_{S} / \alpha_{B}$ ratio, (d) radiation rate $\left(h_{2} / h_{1}\right)$, and (e) the drain temperature $\theta_{\mathrm{f}}$. Volume inflation (from 1 to $5 \mathrm{~cm}$ ) in (a) prolongs the time for reaching the crossing temperature and raises the skin temperature (see inset). (b) The $l_{1}: l_{2}$ ratio has little effect on the relaxation curve. Increasing (c) the $\alpha_{\mathrm{S}} / \alpha_{\mathrm{B}}$ and (d) the $h_{2} / h_{1}$ ratio promotes the Mpemba effect. (e) Lowering the $\theta_{\mathrm{f}}$ shortens the time of the crossing temperature. The sensitivity examination is conducted under conditions of $\alpha_{\mathrm{S}} / \alpha_{\mathrm{B}}=1.48$, $v_{\mathrm{S}}=v_{\mathrm{B}}=10^{-4} \mathrm{~m} \mathrm{~s}^{-1}, \theta_{\mathrm{f}}=0{ }^{\circ} \mathrm{C}, l_{1}=10 \mathrm{~mm}, l_{2}=1 \mathrm{~mm}, h_{1} / \kappa_{\mathrm{B}}=h_{2} / \kappa_{\mathrm{S}}=30 \mathrm{w} \mathrm{m}^{-2} \mathrm{~K}^{-1}$ unless indicated otherwise.

An interplay of the $\mathrm{O}: \mathrm{H}$ van der Waals (vdW-like) force, the $\mathrm{H}-\mathrm{O}$ exchange interaction, the $\mathrm{O}-\mathrm{O}$ inter-electron-pair repulsion (C-repulsion), and the specific heat disparity between the $\mathrm{O}: \mathrm{H}$ and the $\mathrm{H}-\mathrm{O}$ bond dislocate $\mathrm{O}$ atoms always in the same direction along the respective potential paths. ${ }^{22}$ The softer $\mathrm{O}: \mathrm{H}\left(d_{\mathrm{L}}\right)$ nonbond always relaxes more in length than the stiffer $\mathrm{H}-\mathrm{O}\left(d_{\mathrm{H}}\right)$ bond does.

Generally, heating stores energy into a substance by stretching all bonds involved. However, heating stores energy into water by shortening the $\mathrm{H}-\mathrm{O}$ bond and stretching the $\mathrm{O}: \mathrm{H}$ nonbond simultaneously, which is realized by the thermal expansion of the $\mathrm{O}: \mathrm{H}$ nonbond that drags the $\mathrm{H}-\mathrm{O}$ bond by weakening the Coulomb interaction between electron pairs on adjacent oxygen ions (red line linked spheres in Fig. 7). Cooling does oppositely (blue line linked spheres), like suddenly releasing a pair of coupled, highly deformed bungees from different extents of deformation - one is under stretching and the other under compressing, to emit energy at a rate of history dependence. Energy storage and emission of the entire $\mathrm{O}: \mathrm{H}-\mathrm{O}$ bond mainly through the $\mathrm{H}-\mathrm{O}$ relaxation as $E_{\mathrm{L}}(\sim 0.1 \mathrm{eV})$ of the $\mathrm{O}: \mathrm{H}$ nonbond is only $2.5 \%$ of $E_{\mathrm{H}}(\sim 4.0 \mathrm{eV}) .{ }^{14}$ The O:H-O bond memory and the unusual way of energy ejection yield the history-dependent velocity of the $\mathrm{H}-\mathrm{O}$ bond under cooling, given in Fig. $5 \mathrm{~b}$.

Molecular undercoordination effects the same to liquid heating upon the $\mathrm{O}: \mathrm{H}-\mathrm{O}$ bond relaxation, which shortens and stiffens the $\mathrm{H}-\mathrm{O}$ bond and, meanwhile, lengthens and softens the $\mathrm{O}: \mathrm{H}$ nonbond, depressing the local mass density and shifting the frequency of the respective phonons, as summarized in Table 1. Heating and molecular undercoordination enhance each other upon the $\mathrm{O}: \mathrm{H}-\mathrm{O}$ bond relaxation and the associated physical properties in the skin region. The lowered 

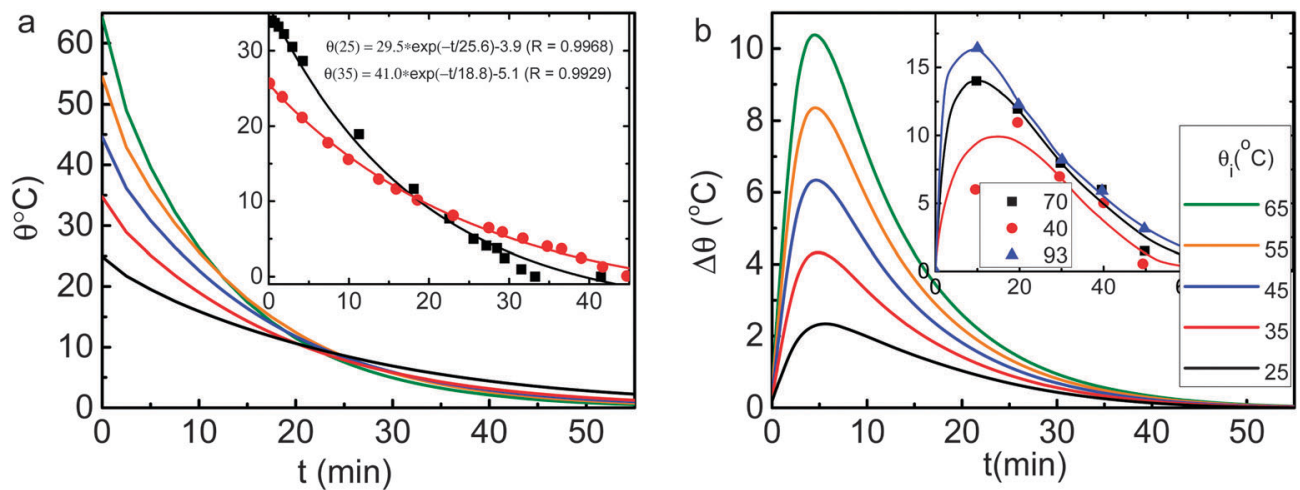

Fig. 4 Numerical reproduction of the measured (insets) (a) thermal relaxation $\theta\left(\theta_{\mathrm{i}}, t\right)$ and (b) skin-bulk temperature difference $\Delta \theta\left(\theta_{\mathrm{i}}, t\right)$ curves ${ }^{2,12}$ for water cooling from different $\theta_{\mathrm{i}}$. Results were obtained using the conditions given in Fig. 3 .
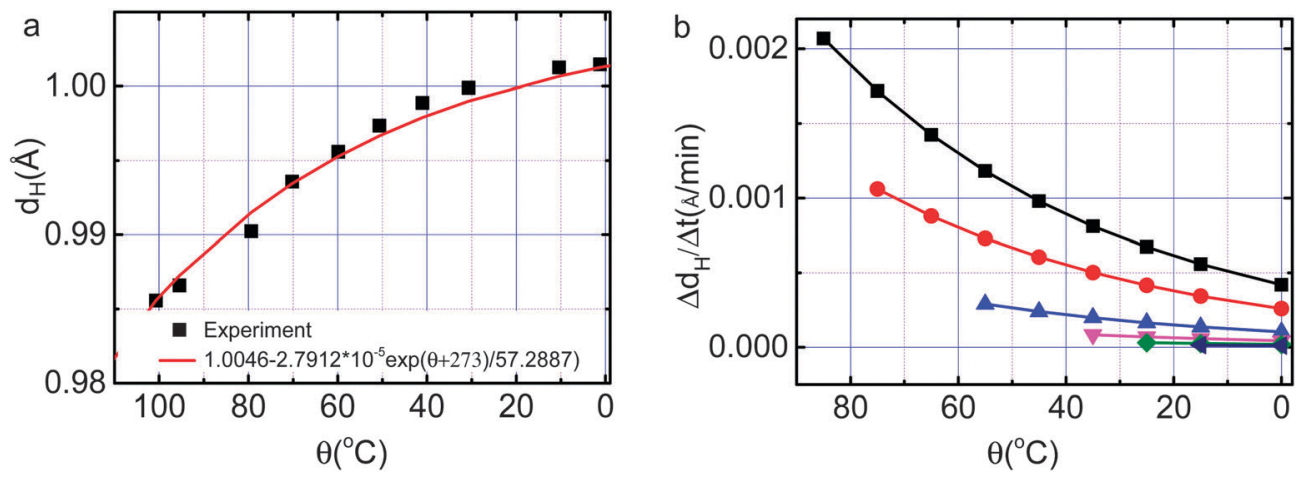

Fig. 5 The (a) measured (scattered data) and simulated (solid line) $d_{\mathrm{H}}(\theta)$ and (b) the experimentally derived $\theta_{\mathrm{i}}$ (corresponds to the starting point of each line) dependence of the $d_{\mathrm{H}}$ velocity during relaxation under cooling. The velocity of the initially shorter $\mathrm{H}-\mathrm{O}$ bond at higher $\theta_{\mathrm{i}}$ remains always higher than those initially longer ones at lower $\theta_{\mathrm{i}}$ values when they meet.
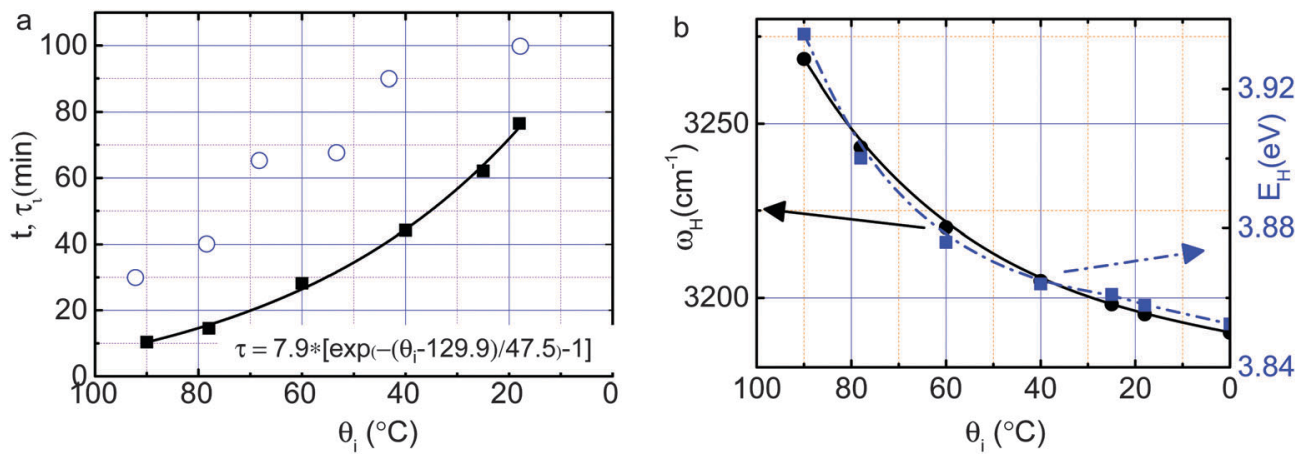

Fig. 6 (a) Cooling time $t$ (scattered circles) dependent relaxation time $\tau_{\mathrm{i}}$ (fitted in solid line) is correlated with the (b) initial energy $E_{\mathrm{H}}$ (solid black line) and vibration frequency $\omega_{\mathrm{H}}$ (dashed blue line) ${ }^{20}$ of the liquid source cooling at different $\theta_{\mathrm{i}}$.

mass density raises the thermal diffusivity (see eqn (1)), which favors heat diffusion outwardly in the conduction path.

\subsection{Source-drain interface: non-adiabatic cycling}

It is necessary to emphasize that the Mpemba effect occurs only under the circumstance that the temperature drops abruptly from $\theta_{\mathrm{i}}$ to $\theta_{\mathrm{f}}$ at the source-drain interface. Fourier solution indicates that the Mpemba crossing temperature is sensitive to the volume of the liquid source (Fig. 3a). Too large liquid volume may prevent this effect by heat-dissipation hindering. As confirmed by Brownridge, $^{7}$ any spatial temperature decay between the source and the drain could prevent the Mpemba effect from occurring. The procedures of decay include tube end sealing, oil film covering, source-drain vacuum isolation, connecting muffin-tin like containers, or putting multiple sources into the limited volume of a fridge. Conducting experiments under identical conditions is necessary to minimize artifacts such as radiation, source/drain volume ratio, exposing area, container material, etc. 


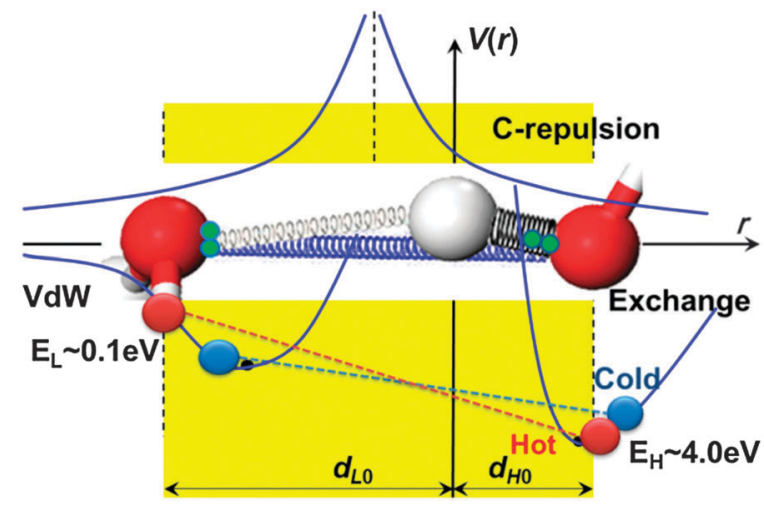

Fig. $7 \mathrm{O}: \mathrm{H}-\mathrm{O}$ bond short-range interactions and the $\mathrm{O}: \mathrm{H}-\mathrm{O}$ (denoted as $d_{\mathrm{L}}$ and $d_{\mathrm{H}}$ ) cooperative relaxation dynamics. ${ }^{23,24} d_{\mathrm{HO}}$ and $d_{\mathrm{LO}}$ are the respective references at $4{ }^{\circ} \mathrm{C}$. Indicated are the van der Waals like (vdW approaches the nonbonding interaction) interaction $\left(E_{\mathrm{L}} \sim 0.1 \mathrm{eV}\right)$ of the $\mathrm{O}: \mathrm{H}$ nonbond (left-handed side), the exchange interaction $\left(E_{\mathrm{H}} \sim 4.0 \mathrm{eV}\right)$ of the $\mathrm{H}-\mathrm{O}$ bond (right-handed side), and the Coulomb repulsion between electron pairs (paring green dots) on oxygen ions. A combination of these interactions and the specific heat disparity between the $\mathrm{O}: \mathrm{H}$ and the $\mathrm{H}-\mathrm{O}$ bond dislocate $\mathrm{O}$ atoms in the same direction by different amounts under cooling. The relaxation proceeds along the $\mathrm{O}: \mathrm{H}-\mathrm{O}$ bond potentials with $\mathrm{H}$ atoms (in grey) being the coordination origin under heating (red line linked spheres, denoted hot) or cooling (blue line linked spheres, denoted cold). Springs of different diameters represent the strengths of the respective interactions.

\subsection{Other factors: supercooling, solutes, and evaporation}

Supercooling is associated with the slower relaxation of the longer $\mathrm{H}-\mathrm{O}$ bond at an initially lower temperature. It has been confirmed that $E_{\mathrm{H}}$ determines the critical temperature for phase transition. ${ }^{22}$ Generally, superheating is associated with the shorter $\mathrm{H}-\mathrm{O}$ bond pertained to water molecules with fewer than four neighbors such as those forming the skin, monolayer film, or droplet on a hydrophobic surface. ${ }^{25}$ Supercooling is associated with the longer $\mathrm{H}-\mathrm{O}$ bond between molecules in contact with the hydrophilic surface ${ }^{26}$ or being compressed. ${ }^{22}$ A $210 \mathrm{MPa}$ compression lowers the melting point to $-22{ }^{\circ} \mathrm{C}$ according to the phase diagram. ${ }^{15}$ The supercooling of the colder water in the Mpemba process ${ }^{7}$ evidences that the initially longer $\mathrm{H}-\mathrm{O}$ bond of colder water is slower than those in the warmer

water to relax at icing because of the slower momentum of the relaxation - memory effect.

The involvement of ionic solutes or impurities ${ }^{27,28}$ mediates the Coulomb coupling because of the alternation of charge quantities and ionic volumes. ${ }^{29,30}$ Salting shares the same effect of heating on the $\mathrm{H}-\mathrm{O}$ phonon blue shift, ${ }^{31,32}$ which is expected to enhance the velocity of heat ejection under cooling. Mass loss due to evaporation of the liquid source ${ }^{3}$ affects the $\mathrm{O}: \mathrm{H}-\mathrm{O}$ relaxation little as the amount of evaporation is negligible under cooling. We have confirmed that the mass loss is only $1.5 \%$ or lower in repeating the experiments by freezing $75{ }^{\circ} \mathrm{C}$ water to $-40{ }^{\circ} \mathrm{C}$ ice.

\section{Conclusion}

Reproduction of observations revealed the following pertaining to the Mpemba paradox:

(1) O:H-O bond possesses memory, whose thermal relaxation defines intrinsically the rate of energy emission. Heating stores energy in water by $\mathrm{O}: \mathrm{H}-\mathrm{O}$ bond deformation. The $\mathrm{H}-\mathrm{O}$ bond is shorter and stiffer in hotter water than in colder water. Cooing does oppositely to emit energy with a thermal momentum that is history dependent.

(2) Heating enhances the skin supersolidity and the skin thermal diffusivity by $\alpha_{\mathrm{S}} / \alpha_{\mathrm{B}} \geq \rho_{\mathrm{B}} / \rho_{\mathrm{S}}=4 / 3$. Convection alone produces no Mpemba effect but only raises the skin temperature slightly.

(3) A highly non-adiabatic ambient system is necessary to ensure the immediate energy dissipation at the source-drain interface. The Mpemba crossing temperature is not only sensitive to the volume of the liquid source but also to the drain temperature and to the radiation rate.

(4) The Mpemba effect takes place with a characteristic relaxation time that drops exponentially with the increase of the initial temperature or the initial energy storage of the liquid.

(5) The O:H-O bond memory may be implicated in living cells in which the hydrogen bond relaxation dominates the signaling, messaging, and damage recovery.

\section{Appendix}
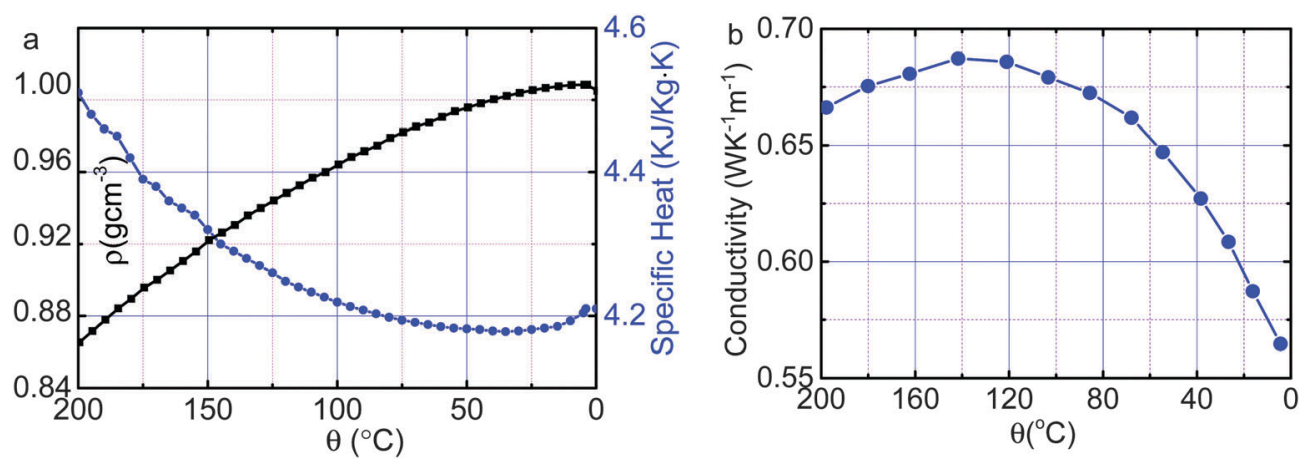

Fig. 8 Temperature dependence ${ }^{33}$ of (a) the mass density $\rho$ (in black) and specific heat $C_{\mathrm{p}}$ (in blue) and (b) the thermal conductivity $\kappa$ (ref. 19) of liquid water, which form the thermal diffusivity of bulk water $\alpha_{\mathrm{B}}(\theta)=\kappa(\theta) /\left[\rho(\theta) C_{\mathrm{p}}(\theta)\right]$. 
Table 1 Summary of the skin supersolidity and the $\mathrm{O}: \mathrm{H}-\mathrm{O}$ bond-electron-phonon attribute under various conditions. Quantities are derived using the following equations ${ }^{16}$ from measurements (indicated with ref.). This equation means that one can derive the lengths of the $\mathrm{O}: \mathrm{H}$ nonbond and the $\mathrm{H}-\mathrm{O}$ bond with the measured mass density under applied stimulus such as molecules with fewer coordination neighbors ${ }^{14}$ or heating ${ }^{34}$ (see Fig. $5 a$ for instance $)^{a}$ :

$$
\text { (a) }
$$$$
\begin{cases}d_{\mathrm{OO}}=2.6950 \rho^{-1 / 3} & (\text { Molecular separation }) \\ d_{\mathrm{L}}=\frac{2 d_{\mathrm{L} 0}}{1+\exp \left[\left(d_{\mathrm{H}}-d_{\mathrm{H} 0}\right) / 0.2428\right]} ; & \left(d_{\mathrm{H} 0}=1.0004, d_{\mathrm{L} 0}=1.6946\right)\end{cases}
$$

\begin{tabular}{|c|c|c|c|c|}
\hline & \multicolumn{2}{|c|}{ Water $(298 \mathrm{~K})$} & \multirow{2}{*}{$\frac{\text { Ice }(253 \mathrm{~K})}{\text { Bulk }}$} & \multirow{2}{*}{$\frac{\text { Vapor }}{\text { Monomer }}$} \\
\hline & Bulk & Skin & & \\
\hline O1s binding energy $E_{1 \mathrm{~s}}(\mathrm{eV})^{35}$ & 536.6 & 538.1 & - & 539.7 \\
\hline $\mathrm{H}-\mathrm{O}$ bond energy $E_{\mathrm{H}}(\mathrm{eV})$ & $3.97^{21}$ & $4.52-4.66$ & - & $5.10^{36}$ \\
\hline Melting point $T_{\mathrm{m}}(\mathrm{K})$ & 273 & $315^{37}$ & 273 & \\
\hline $\mathrm{H}-\mathrm{O}$ phonon frequency $\omega_{\mathrm{H}}\left(\mathrm{cm}^{-1}\right)^{38}$ & 3200 & 3450 & 3125 & $3650^{39}$ \\
\hline $\mathrm{O}: \mathrm{H}$ phonon frequency $\omega_{\mathrm{L}}\left(\mathrm{cm}^{-1}\right)^{34}$ & 220 & $\sim 180^{14}$ & 210 & - \\
\hline $\mathrm{H}-\mathrm{O}$ bond length $d_{\mathrm{H}}(\AA)^{16}$ & 0.9981 & 0.8406 & 0.9676 & 0.8030 \\
\hline $\mathrm{O}: \mathrm{H}$ nonbond length $d_{\mathrm{L}}(\AA)^{16}$ & 1.6969 & 2.1126 & 1.8034 & $\geq 2.177$ \\
\hline $\mathrm{O}-\mathrm{O}$ distance $d_{\mathrm{OO}}(\AA)$ & $2.700^{40}$ & $2.965^{41}$ & $2.771^{16}$ & $2.980^{41}$ \\
\hline Mass density $\rho\left(\mathrm{g} \mathrm{cm}^{-3}\right)^{16}$ & 0.9945 & 0.7509 & $0.92^{42}$ & $\leq 0.7396$ \\
\hline
\end{tabular}

${ }^{a}$ The phonon frequency shift is proportional to the square of bond stiffness: $\Delta \omega \propto \sqrt{E d^{-2}} \propto \sqrt{Y d}$ with $Y$ being the elastic modulus that is proportional to the energy density $E d^{-3} \cdot{ }^{29}$

\section{Acknowledgements}

Critical reading by Yi Sun, assistance in confirming the mass loss $(<1.5 \%)$ during Mpemba experiments by Lei Li, Hongwei Tian, and Xiaoming Zhang, and financial support from NSF (No. 21273191, 11274198, and 51102148) China and MOE (RG29/12) Singapore are gratefully acknowledged.

\section{References}

1 Aristotle, Meteorology 350 B.C.E: http://classics.mit.edu/Aris totle/meteorology.1.i.html.

2 E. B. Mpemba and D. G. Osborne, Cool?, Phys. Educ., 1979, 14, 410-413.

3 D. Auerbach, Supercooling and the Mpemba effect - when hot-water freezes quicker than cold, Am. J. Phys., 1995, 63(10), 882-885.

4 M. Jeng, The Mpemba effect: when can hot water freeze faster than cold?, Am. J. Phys., 2006, 74(6), 514.

5 C. A. Knight, The Mpemba effect: the freezing times of hot and cold water, Am. J. Phys., 1996, 64(5), 524.

6 M. Vynnycky and S. L. Mitchell, Evaporative cooling and the Mpemba effect, Heat Mass Transfer, 2010, 46(8-9), 881-890.

7 J. D. Brownridge, When does hot water freeze faster then cold water? A search for the Mpemba effect, Am. J. Phys., 2011, 79(1), 78.

8 J. I. Katz, When hot water freezes before cold, Am. J. Phys., 2009, 77(1), 27-29.

9 P. Ball, Does hot water freeze first, Phys. World, 2006, 19(4), 19-21.

10 H. Heffner, The Mpemba effect: http://www.mtaonline. net/ hheffner/Mpemba.pdf, 2001.

11 M. Vynnycky and N. Maeno, Axisymmetric natural convectiondriven evaporation of hot water and the Mpemba effect, Int. J. Heat Mass Transfer, 2012, 55(23-24), 7297-7311.
12 N. Bregović, Mpemba effect from a viewpoint of an experimental physical chemist, http://www.rsc.org/images/nikola-bregovicentry_tcm18-225169.pdf, 2012.

13 L. B. Kier and C. K. Cheng, Effect of Initial Temperature on Water Aggregation at a Cold Surface, Chem. Biodiversity, 2013, 10(1), 138-143.

14 C. Q. Sun, X. Zhang, J. Zhou, Y. Huang, Y. Zhou and W. Zheng, Density, Elasticity, and Stability Anomalies of Water Molecules with Fewer than Four Neighbors, J. Phys. Chem. Lett., 2013, 4, 2565-2570.

15 X. Zhang, Y. Huang, Z. Ma, J. Zhou, Y. Zhou, W. Zheng and C. Q. Sun, A common superslid skin covering both water and ice, Phys. Chem. Chem. Phys., 2014, DOI: 10.1039/ C1034CP02516D.

16 Y. Huang, X. Zhang, Z. Ma, Y. Zhou, J. Zhou, W. Zheng and C. Q. Sun, Size, separation, structure order, and mass density of molecules packing in water and ice, Sci. Rep., 2013, 3, 3005.

17 J. Fourier, The Analytical Theory of Heat, Dover Publications, New York, 1955.

18 J. R. Welty, C. E. Wicks, R. E. Wilson and G. L. Rorrer, Fundamentals of Momentum, Heat and Mass transfer, John Wiley and Sons, 2007.

19 Water Thermal Properties - The Engineering Toolbox [Online], available: http://www.engineeringtoolbox.com/waterthermal-properties-d_162.html.

20 P. C. Cross, J. Burnham and P. A. Leighton, The Raman spectrum and the structure of water, J. Am. Chem. Soc., 1937, 59, 1134-1147.

21 Y. Huang, X. Zhang, Z. Ma, Y. Zhou, G. Zhou and C. Q. Sun, Hydrogen-bond asymmetric local potentials in compressed ice, J. Phys. Chem. B, 2013, 117(43), 13639-13645.

22 C. Q. Sun, X. Zhang and W. T. Zheng, Hidden force opposing ice compression, Chem. Sci., 2012, 3, 1455-1460.

23 G. R. Desiraju, A Bond by Any Other Name, Angew. Chem., Int. Ed., 2011, 50(1), 52-59. 
24 L. R. Falvello, The Hydrogen Bond, Front and Center, Angew. Chem., Int. Ed., 2010, 49(52), 10045-10047.

25 A. Uysal, M. Chu, B. Stripe, A. Timalsina, S. Chattopadhyay, C. M. Schlepütz, T. J. Marks and P. Dutta, What x rays can tell us about the interfacial profile of water near hydrophobic surfaces, Phys. Rev. B: Condens. Matter Mater. Phys., 2013, 88(3), 035431.

26 F. G. Alabarse, J. Haines, O. Cambon, C. Levelut, D. Bourgogne, A. Haidoux, D. Granier and B. Coasne, Freezing of Water Confined at the Nanoscale, Phys. Rev. Lett., 2012, 109(3), 035701.

27 M. Freeman, Cooler Still, Phys. Educ., 1979, 14, 417-421.

28 B. Wojciechowski, Freezing of Aqueous Solutions Containing Gases, Cryst. Res. Technol., 1988, 23, 843-848.

29 C. Q. Sun, Relaxation of the Chemical Bond, Springer Ser. Chem. Phys., vol. 108, Springer Press, Berlin, 2014, p. 550, ISBN: 978-981-4585-20-0.

30 J. D. Smith, R. J. Saykally and P. L. Geissler, The Effects of Dissolved Halide Anions on Hydrogen Bonding in Liquid Water, J. Am. Chem. Soc., 2007, 129, 13847-13856.

31 Q. Sun, Raman spectroscopic study of the effects of dissolved $\mathrm{NaCl}$ on water structure, Vib. Spectrosc., 2012, 62, 110-114.

32 S. Park and M. D. Fayer, Hydrogen bond dynamics in aqueous $\mathrm{NaBr}$ solutions, Proc. Natl. Acad. Sci. U. S. A., 2007, 104(43), 16731-16738.

33 M. Chaplin. Water structure and science: http://www.lsbu. ac.uk/water/.

34 C. Q. Sun, X. Zhang, X. Fu, W. Zheng, J.-l. Kuo, Y. Zhou, Z. Shen and J. Zhou, Density and phonon-stiffness anomalies of water and ice in the full temperature range, J. Phys. Chem. Lett., 2013, 4, 3238-3244.
35 M. Abu-Samha, K. J. Borve, M. Winkler, J. Harnes, L. J. Saethre, A. Lindblad, H. Bergersen, G. Ohrwall, O. Bjorneholm and S. Svensson, The local structure of small water clusters: imprints on the core-level photoelectron spectrum, J. Phys. B: At., Mol. Opt. Phys., 2009, 42(5), 055201.

36 S. A. Harich, D. W. H. Hwang, X. Yang, J. J. Lin, X. Yang and R. N. Dixon, Photodissociation of $\mathrm{H}_{2} \mathrm{O}$ at $121.6 \mathrm{~nm}$ : A stateto-state dynamical picture, J. Chem. Phys., 2000, 113(22), 10073-10090.

37 H. Qiu and W. Guo, Electromelting of Confined Monolayer Ice, Phys. Rev. Lett., 2013, 110(19), 195701.

38 T. F. Kahan, J. P. Reid and D. J. Donaldson, Spectroscopic probes of the quasi-liquid layer on ice, J. Phys. Chem. A, 2007, 111(43), 11006-11012.

39 Y. R. Shen and V. Ostroverkhov, Sum-frequency vibrational spectroscopy on water interfaces: polar orientation of water molecules at interfaces, Chem. Rev., 2006, 106(4), 1140-1154.

40 U. Bergmann, A. Di Cicco, P. Wernet, E. Principi, P. Glatzel and A. Nilsson, Nearest-neighbor oxygen distances in liquid water and ice observed by x-ray Raman based extended x-ray absorption fine structure, J. Chem. Phys., 2007, 127(17), 174504.

41 K. R. Wilson, R. D. Schaller, D. T. Co, R. J. Saykally, B. S. Rude, T. Catalano and J. D. Bozek, Surface relaxation in liquid water and methanol studied by x-ray absorption spectroscopy, J. Chem. Phys., 2002, 117(16), 7738-7744.

42 F. Mallamace, M. Broccio, C. Corsaro, A. Faraone, D. Majolino, V. Venuti, L. Liu, C. Y. Mou and S. H. Chen, Evidence of the existence of the low-density liquid phase in supercooled, confined water, Proc. Natl. Acad. Sci. U. S. A., 2007, 104(2), 424-428. 